\title{
AN IMPLICIT ONE-STEP THIRD DERIVATIVE HYBRID BLOCK METHOD FOR THE NUMERICAL SOLUTION OF FIRST ORDER INITIAL VALUE PROBLEMS IN ORDINARY DIFFERENTIAL EQUATIONS.
}

\author{
Ononogbo , .C. B. ${ }^{1}$, Airemen, .I. E. ${ }^{2}$, Julia, .U. E. ${ }^{3}$ and Ishie, .I.H. ${ }^{4}$ \\ (Department of Mathematics, Ambrose Alli University Ekpoma, Edo State, Nigeria) 1,2 \\ (Department of Basic Sciences school of General Studies, Auchi Polytechnic,Auchi Edo State, Nigeria) ${ }^{3}$ \\ (Department of General studies, Bellacks Polytechnic Kwale, Delta State, Nigeria) ${ }^{4}$
}

\begin{abstract}
ABSRACT: In this article, an implicit one-step third derivative hybrid block method was developed for the numerical solution of first order initial value problems in ordinary differential Equations. In developing the method, interpolation and collocation techniques were considered for developing hybrid block methods. In developing the method we employed the conventional Taylor series expansion. To further justify the usability of the proposed method, the basic properties which infer convergence when adopted to solve ordinary differential equations were investigated. The proposed method validates its superiority over existing methods as seen in the accuracy of our results from the numerical examples. Furthermore, we called this formula OCB12 method
\end{abstract}

Keywords: Hybrid Block Method, First Order, Initial Value Problems, Taylor Series, Collocation, Interpolation, Third derivative.

\section{INTRODUCTION}

In this article, we proposed an implicit one-step third derivative hybrid block method for solving first order initial value problems in ordinary differential equations of the form

$y^{\prime}=f(x, y), \quad y\left(x_{0}\right)=y_{0}, \quad a \leq x \leq b$

where $x_{0}$ is the initial point, $y_{0}$ is the solution at the initial point and $f$ is assumed to satisfy Lipchitz condition as stated below without proof.

Theorem 1[1]: let $f(x, y)$ be defined and continuous for all points $(x, y)$ in the region $\mathrm{D}$ defined by $a \leq x \leq b,-\infty<$ $y<\infty, a$ and $b$ finite, and let there exist a constant $L$ such that, for every, $x, y, y^{*},(x, y)$ and $\left(x, y^{*}\right)$ are both in D;

$\left|f(x, y)-f\left(x, y^{*}\right)\right| \leq L\left|y-y^{*}\right|$

Then, if $y_{0}$ is any given number, there exist a unique solution $y(x)$ of the initial value problem (1), where $y(x)$ is continuous and differentiable for all $(x, y)$ in $D$. According to [2], equation (1) is used to model problems involving trajectory of a ballistic missile, the motion of an artificial satellite in its orbit, theories concerning electrical networking, bending of beams, stability of aircraft and many others. Most often, these problems do not have analytical solutions; hence appropriate numerical methods are adopted to solve such problems [1]. Some approaches to numerical method 


\section{International Advanced Research Journal in Science, Engineering and Technology}

Vol. 8, Issue 8, August 2021

DOI: $10.17148 /$ IARJSET.2021.8825

include the Runge Kutta type, (self-starting), the linear multistep methods, particularly the implicit methods, and many others [3]. Conventionally, implicit linear multistep methods are implemented in the predictor-corrector mode which is prone to error propagation as the integration process progresses. The disadvantages associated with the predictorcorrector method led to the development of block methods from linear multistep method. Block methods which are used by many scholars for solving problem (1), were first introduced by [4] and later by [5] mainly to provide starting values for predictor-corrector algorithms. Those methods produced better accuracy than the usual step by step methods. [6], on the other hand, extended Milne's idea to develop block methods for solving ODEs. In order to obtain higher order methods and increase the accuracy of the approximate solution, [7] proposed hybrid block methods which included off-step point(s) in the derivation of the algorithms. Furthermore, hybrid block methods were used to circumvent Dahlquists barrier conditions, which stipulate that the order of a k-step Linear Multistep Method (LMM) cannot exceed $\mathrm{k}+1$ when $\mathrm{k}$ is odd or $\mathrm{k}+2$ when $\mathrm{k}$ is even for the method to be zero-stable. In addition, hybrid block methods are also known to share with Runge-Kutta methods their favorable advantage of being self starting and more accurate since they are implemented as a block. In hybrid block methods, step and off-step points are combined to form a single block for solving ODEs. In addition, [10] proposed, one and two-step new hybrid methods for the numerical solution of first order initial value problems, [8] proposed, Optimization of one step block method with three hybrid points for solving first-order ordinary differential equations, [9] proposed Extended One-Step Block Method for Solving Stiff equations to enhance the accuracy of the approximation of initial value problems. The main idea of this study is to improve on the work of $[8,9,10]$ by developing an implicit one-step third derivative hybrid block method for numerical solution of first order initial value problems in ordinary differential equations with high accuracy.

\section{DEVELOPMENT OF OCB12 METHOD.}

This section shows the development of the implicit one-step third derivative hybrid block method for solving initial value problems. The scheme for the hybrid block method is developed from the Obrechkoff method given as:

$y_{n+k}=\sum_{j=0}^{k-1} \alpha_{j} y_{n+j}+\sum_{i=1}^{l} h^{i} \sum_{i=0}^{k}\left(\varphi_{\eta i} f_{n+i}+\varphi_{\eta i} g_{n+i}+\varphi_{\eta i} p_{n+i}\right), \eta=1,2,3, k=1, l=3$

In order to obtain the main method for one-step, we rewrite equation (3) to be:

$$
\begin{aligned}
& y_{n+1}=\alpha_{0} y_{0}+h\left(\varphi_{10} y_{n}^{\prime}+\varphi_{1 \frac{1}{3}} y_{n+\frac{1}{3}}^{\prime}+\varphi_{1 \frac{2}{3}} y_{n+\frac{2}{3}}^{\prime}+\varphi_{11} y_{n+1}^{\prime}\right)+h^{2}\left(\varphi_{20} y_{n}^{\prime \prime}+\varphi_{2 \frac{1}{3}} y_{n+\frac{1}{3}}^{\prime \prime}+\varphi_{2 \frac{2}{3}} y_{n+\frac{2}{3}}^{\prime \prime}+\varphi_{21} y_{n+1}^{\prime \prime}\right) \\
& +h^{3}\left(\varphi_{30} y_{n}^{\prime \prime \prime}+\varphi_{3 \frac{1}{3}} y_{n+\frac{1}{3}}^{\prime \prime \prime}+\varphi_{3 \frac{2}{3}} y_{n+\frac{2}{3}}^{\prime \prime \prime}+\varphi_{31} y_{n+1}^{\prime \prime \prime}\right)
\end{aligned}
$$

Using Taylor series expansion to expand individual terms in (4) and upon substitution of the expansions back in (4), the matrix form $A x=B$ obtained from the expression:

$y_{n+a}=y\left(x_{n}+a h\right)=y\left(x_{n}\right)+a h y^{\prime}\left(x_{n}\right)+\frac{(a h)^{2}}{2 !} y^{\prime \prime}\left(x_{n}\right)+\frac{(a h)^{3}}{3 !} y^{\prime \prime \prime}\left(x_{n}\right)+\cdots$ and the coefficients of $y^{(m)}\left(x_{n}\right)$ are equated to be: 
International Advanced Research Journal in Science, Engineering and Technology

Vol. 8, Issue 8, August 2021

DOI: 10.17148/IARJSET.2021.8825

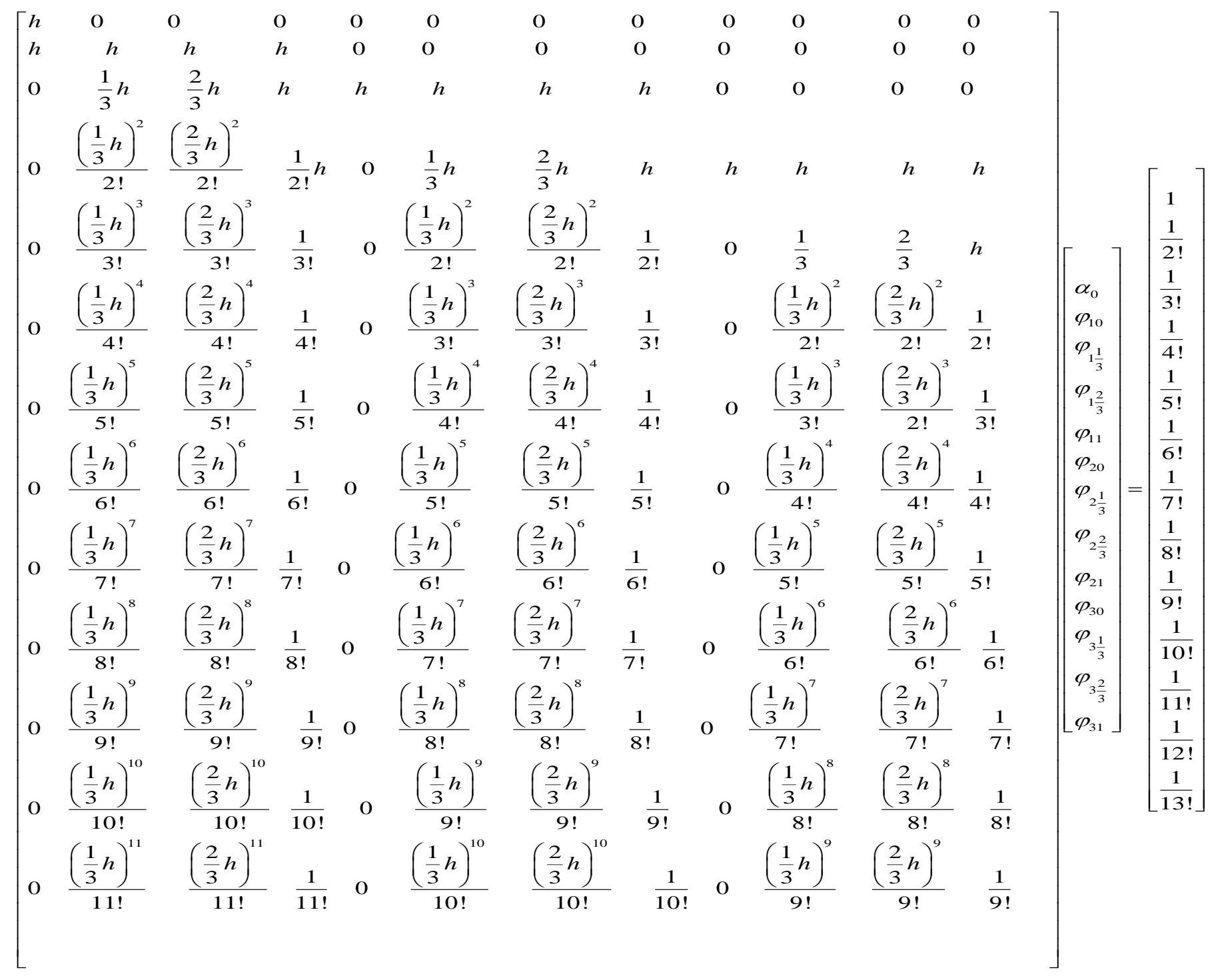

Solving equation (5) we obtained the values of $\left[\alpha_{0}, \varphi_{10}, \varphi_{1 \frac{1}{3}}, \varphi_{1 \frac{2}{3}}, \varphi_{11}, \varphi_{20}, \varphi_{2 \frac{1}{3}}, \varphi_{2 \frac{2}{3}}, \varphi_{21}, \varphi_{30}, \varphi_{3 \frac{1}{3}}, \varphi_{3 \frac{2}{3}}, \varphi_{31}\right]^{T}$ as:

$\left[1, \frac{1283}{9856}, \frac{3645}{9856}, \frac{3645}{9856}, \frac{1283}{9856}, \frac{311}{49280},-\frac{243}{49280}, \frac{243}{49280},-\frac{311}{49280}, \frac{17}{147840}, \frac{81}{49280}, \frac{81}{49280}, \frac{17}{147840}\right]^{T}$

substituting into equation (4) we have:

$$
\begin{aligned}
y_{n+1}= & y_{n}+\frac{1283}{9856} h f_{n}+\frac{3645}{9856} f_{n+\frac{1}{3}}+\frac{3645}{9856} h f_{n+\frac{2}{3}}+\frac{1283}{9856} f_{n+1}+\frac{311}{49280} h^{2} g_{n}-\frac{243}{49280} h^{2} g_{n+\frac{1}{3}}+\frac{243}{49280} h^{2} g_{n+\frac{2}{3}} \\
& -\frac{311}{49280} h^{2} g_{n+1}+\frac{17}{147840} h^{3} p_{n}+\frac{81}{49280} h^{3} p_{n+\frac{1}{3}}+\frac{81}{49280} h^{3} p_{n+\frac{2}{3}}+\frac{17}{147840} h^{3} p_{n+1}
\end{aligned}
$$




\section{International Advanced Research Journal in Science, Engineering and Technology}

Vol. 8, Issue 8, August 2021

DOI: $10.17148 /$ IARJSET.2021.8825

To implement OCB12 method derived in Equation (6), an additional method is needed. This method is obtain by similar approach to have the following:

$$
\begin{aligned}
& y_{n+\frac{1}{3}}=y_{n}+\frac{912523}{7185024} h f_{n}+\frac{23717}{88704} h f_{n+\frac{1}{3}}-\frac{5851}{88704} h f_{n+\frac{2}{3}}+\frac{35339}{7185024} h f_{n+1}+\frac{214943}{35925120} h^{2} g_{n}-\frac{10657}{1330560} h^{2} g_{n+\frac{1}{3}} \\
& +\frac{10657}{1330560} h^{2} g_{n+\frac{2}{3}}-\frac{5941}{11975040} h^{2} g_{n+1}+\frac{11369}{107775360} h^{3} p_{n}+\frac{4423}{2395008} h^{3} p_{n+\frac{1}{3}}-\frac{7453}{11975040} h^{3} p_{n+\frac{2}{3}}+\frac{1513}{107775360} h^{3} p_{n+1}
\end{aligned}
$$

$$
\begin{aligned}
& y_{n+\frac{2}{3}}=y_{n}+\frac{7031}{56133} h f_{n}+\frac{302}{693} f_{n+\frac{1}{3}}+\frac{71}{693} h f_{n+\frac{2}{3}}+\frac{178}{56133} f_{n+1}+\frac{544}{93555} h^{2} g_{n}+\frac{32}{10395} h^{2} g_{n+\frac{1}{3}} \\
& -\frac{32}{10395} h^{2} g_{n+\frac{2}{3}}-\frac{92}{280665} h^{2} g_{n+1}+\frac{17}{168399} h^{3} p_{n}+\frac{212}{93555} h^{3} p_{n+\frac{1}{3}}-\frac{19}{93555} h^{3} p_{n+\frac{2}{3}}+\frac{8}{841995} h^{3} p_{n+1}
\end{aligned}
$$

Hence, equation (6), (7) and (8) are the required block method for the solution of equation (1)

\subsection{Analysis of OCB12 method}

\subsection{Order of OCB12 method}

We define a linear operator $\mathrm{L}$ by:

$$
L[y(x): h]=\sum_{j=0}^{k}\left[\alpha_{j} y\left(x_{n}+j h\right)-h \varphi_{j} y^{\prime}\left(x_{n}+j h\right)\right]
$$

Where $y(x)$ is an arbitrary test function that is continuously differentiable in the interval $[a, b]$.Expanding $y\left(x_{n}+j h\right)$ and $y^{\prime}\left(x_{n}+j h\right)$ by Taylor's series about the point $x_{n} \quad$ and collecting like terms in $\mathrm{h}$ and $\mathrm{y}$ we obtain:

$$
L[y(x): h]=C_{0} y(x)+C_{1} h y^{\prime}(x)+C_{2} h^{2} y^{\prime \prime}(x)+\ldots+C_{p} h^{p} y^{p}(x)
$$

\section{Definition 3.1}

According to [11], the differential equation (9) and the associated Linear Multistep Method are said to be of order p if

$$
C_{0}=C_{1}=C_{2}=\ldots C_{p}=0, C_{p+1} \neq 0
$$

\section{Definition 3.2}

The term $C_{p+1}$ is called error constant and it implies that the local truncation error is given by

$$
E_{p+1}=C_{p+1} h^{p+1} y^{\left(p^{p+1}\right)}\left(x_{n}\right)+O\left(h^{p+2}\right)
$$

Following Definition 3.1 and 3.2. The order of the block method in equation (6), (7) and (8) is gotten by: 
International Advanced Research Journal in Science, Engineering and Technology

Vol. 8, Issue 8, August 2021

DOI: 10.17148/IARJSET.2021.8825

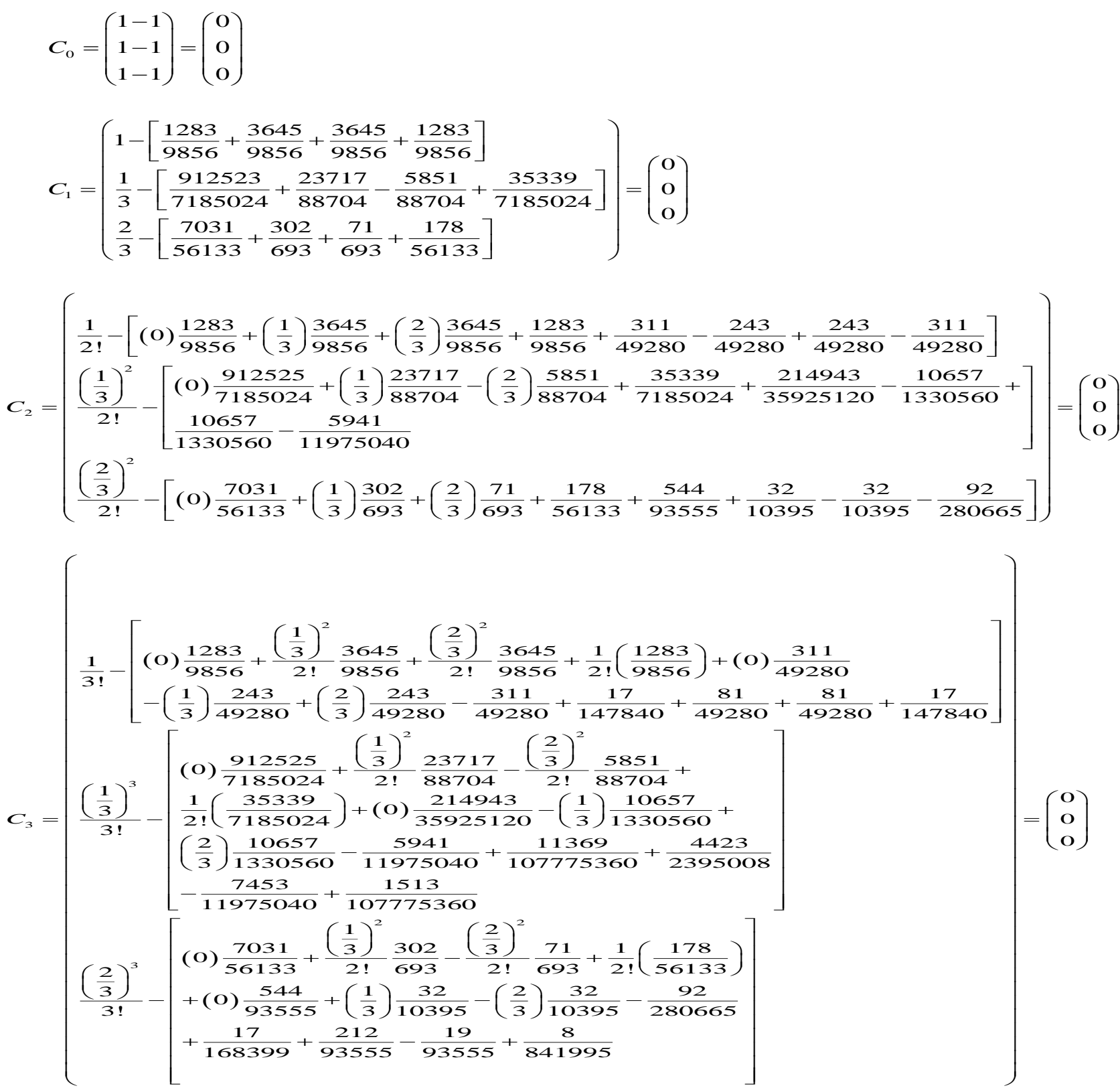


International Advanced Research Journal in Science, Engineering and Technology

Vol. 8, Issue 8, August 2021

DOI: 10.17148/IARJSET.2021.8825

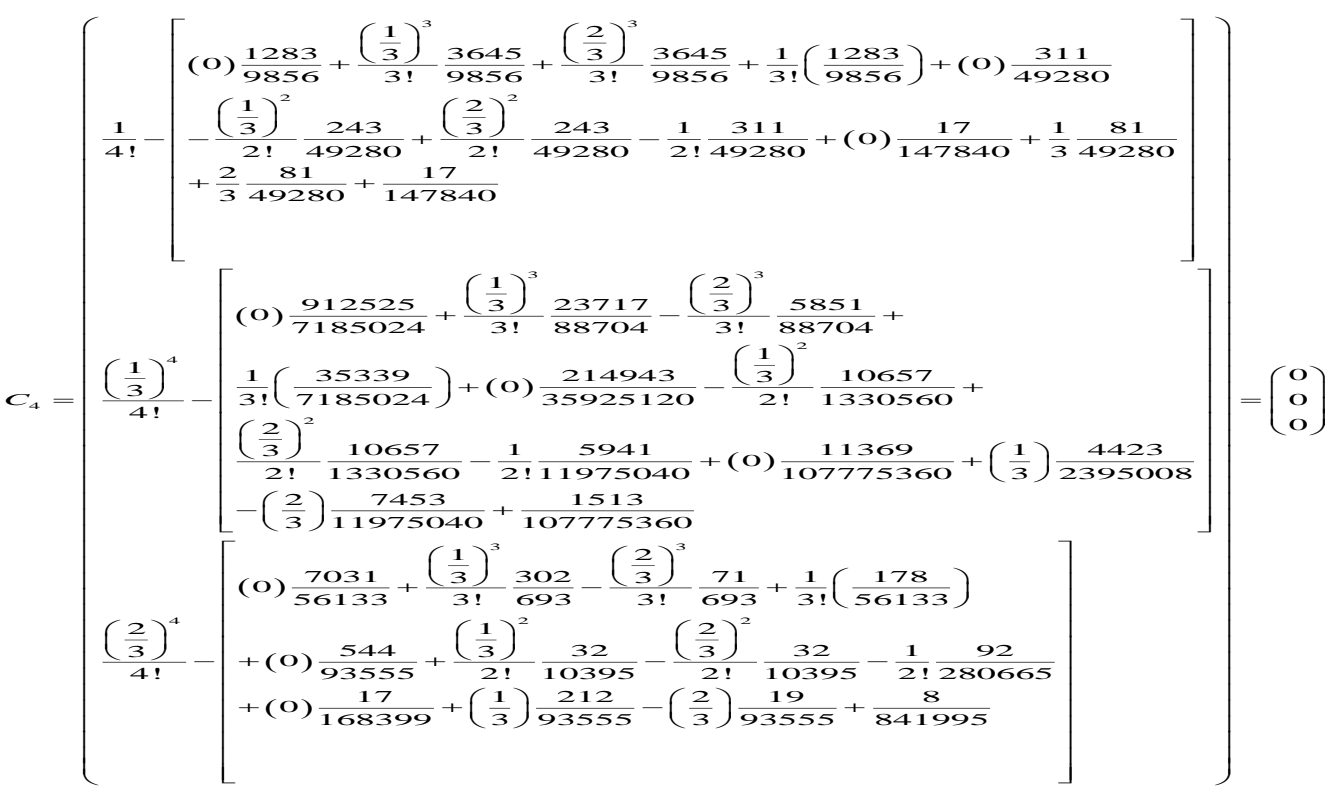

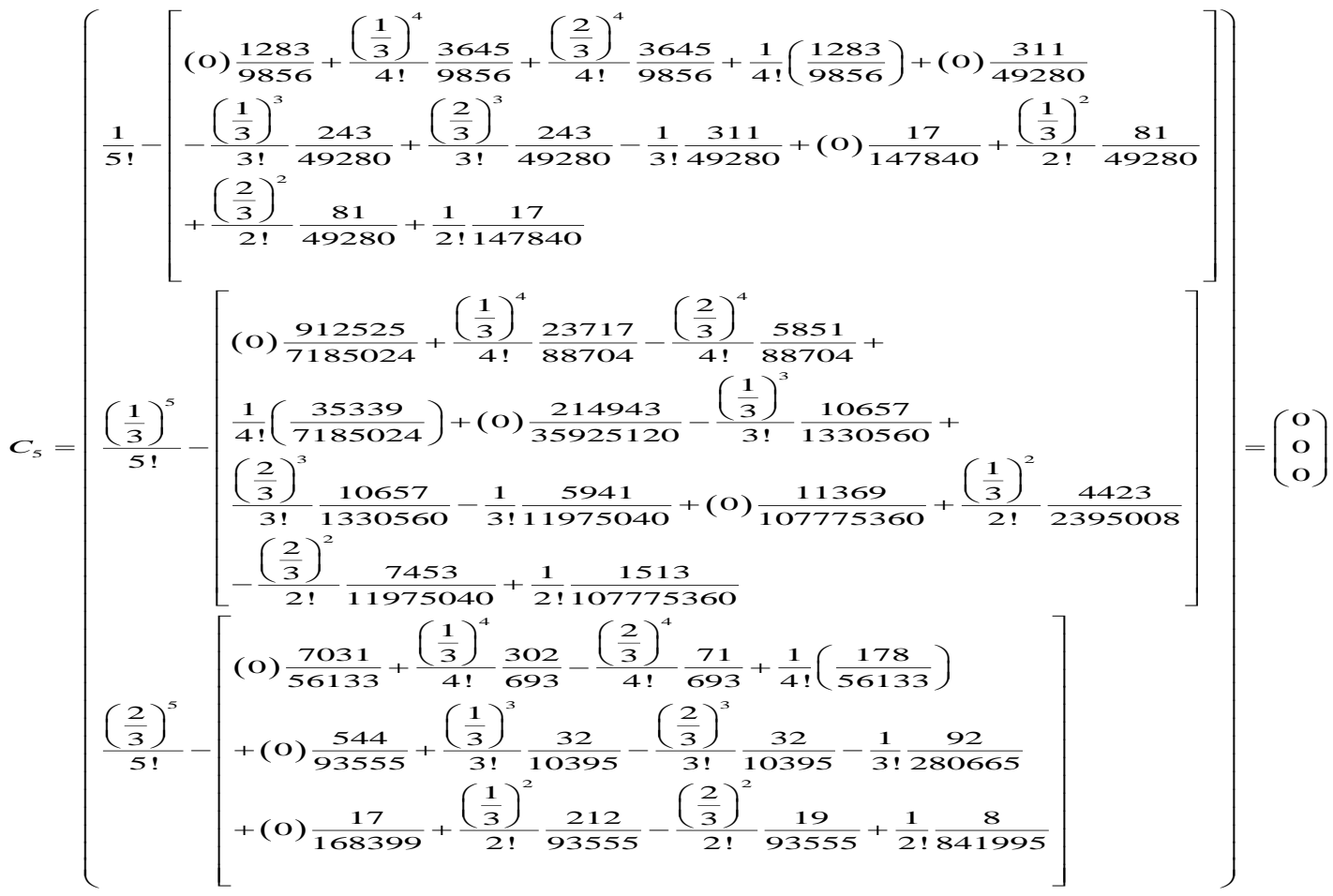


International Advanced Research Journal in Science, Engineering and Technology

Vol. 8, Issue 8, August 2021

DOI: $10.17148 / I A R J S E T .2021 .8825$

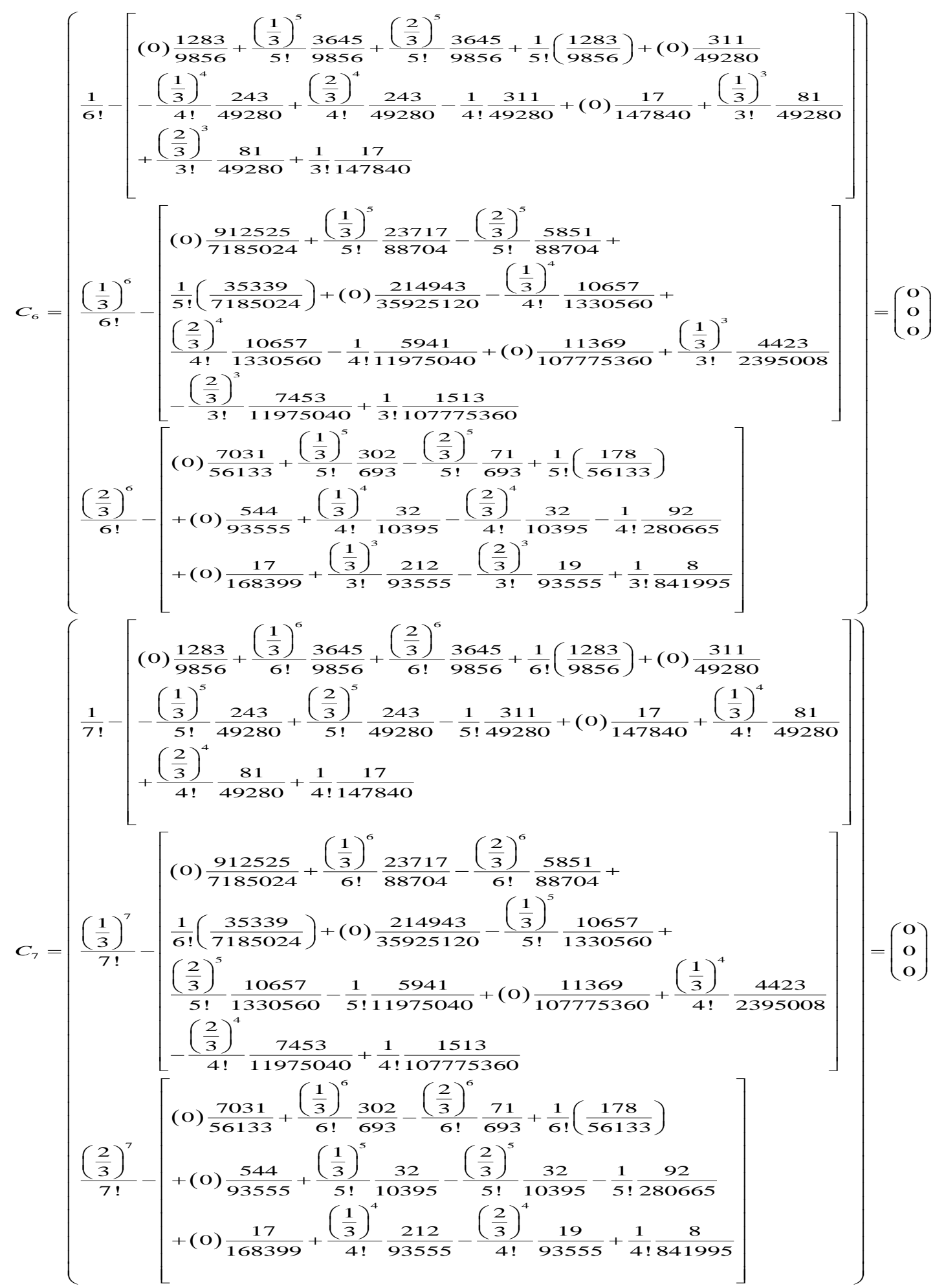


International Advanced Research Journal in Science, Engineering and Technology

Vol. 8, Issue 8, August 2021

DOI: 10.17148/IARJSET.2021.8825

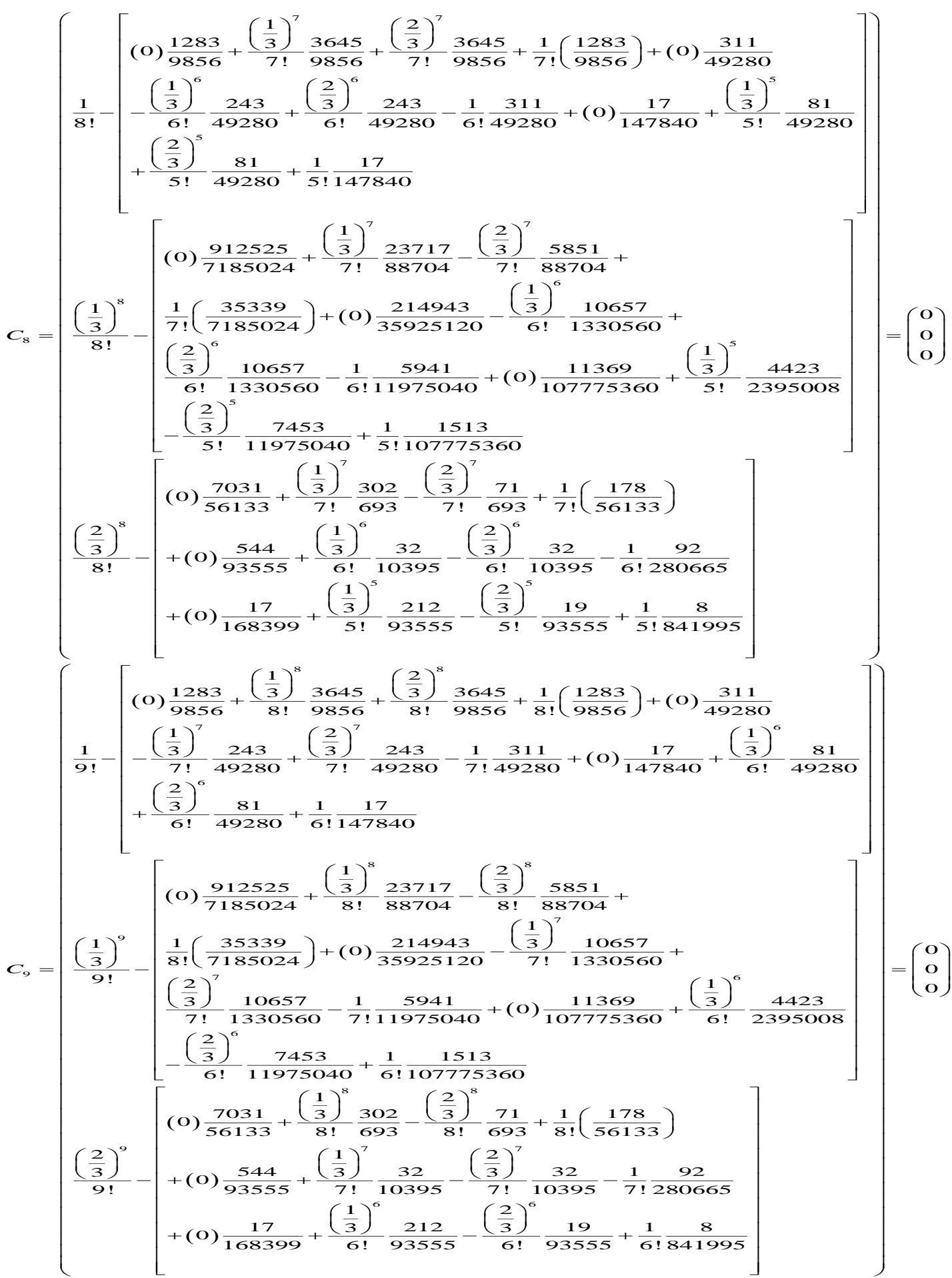


International Advanced Research Journal in Science, Engineering and Technology

Vol. 8, Issue 8, August 2021

DOI: 10.17148/IARJSET.2021.8825

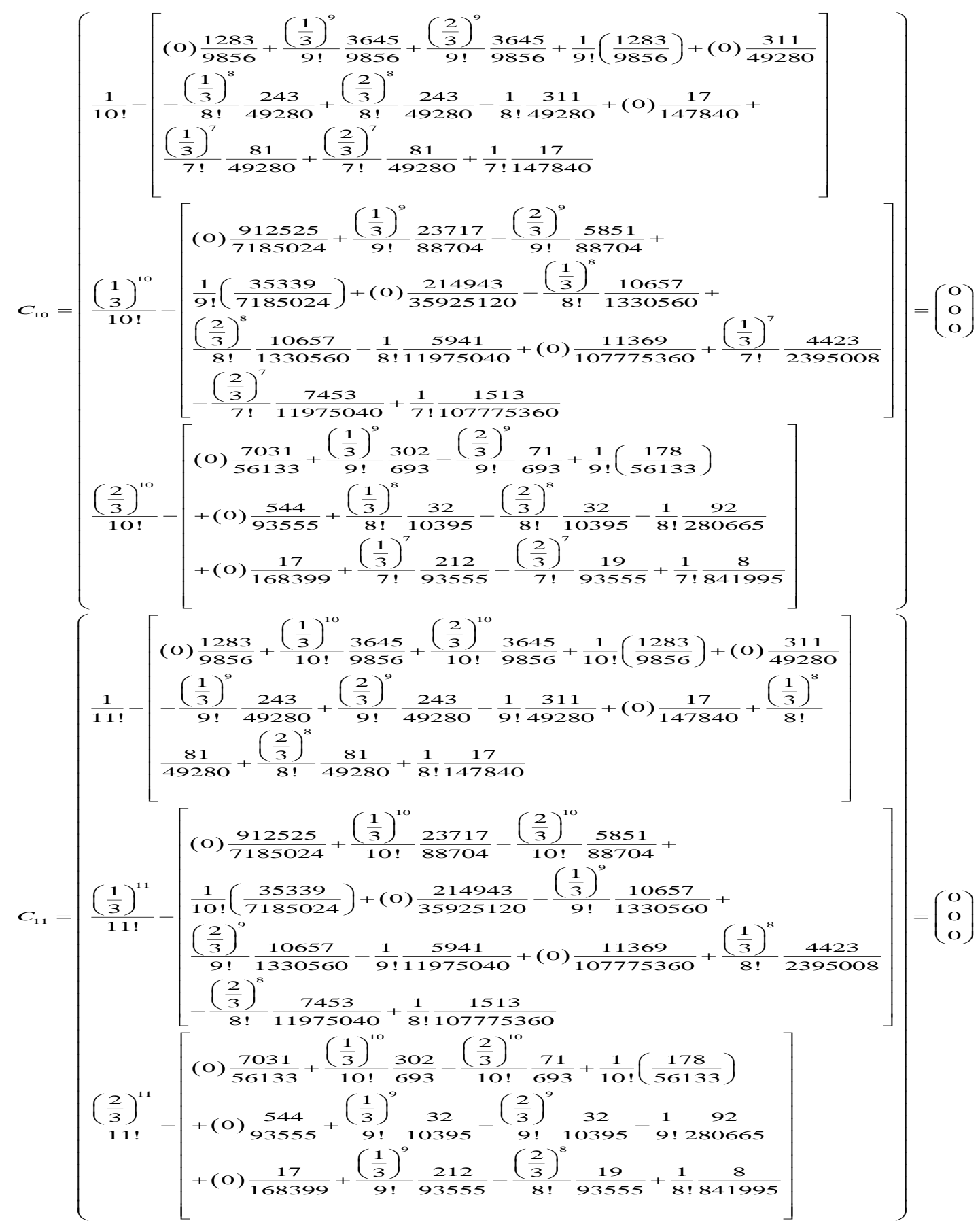


International Advanced Research Journal in Science, Engineering and Technology

Vol. 8, Issue 8, August 2021

DOI: $10.17148 /$ IARJSET.2021.8825

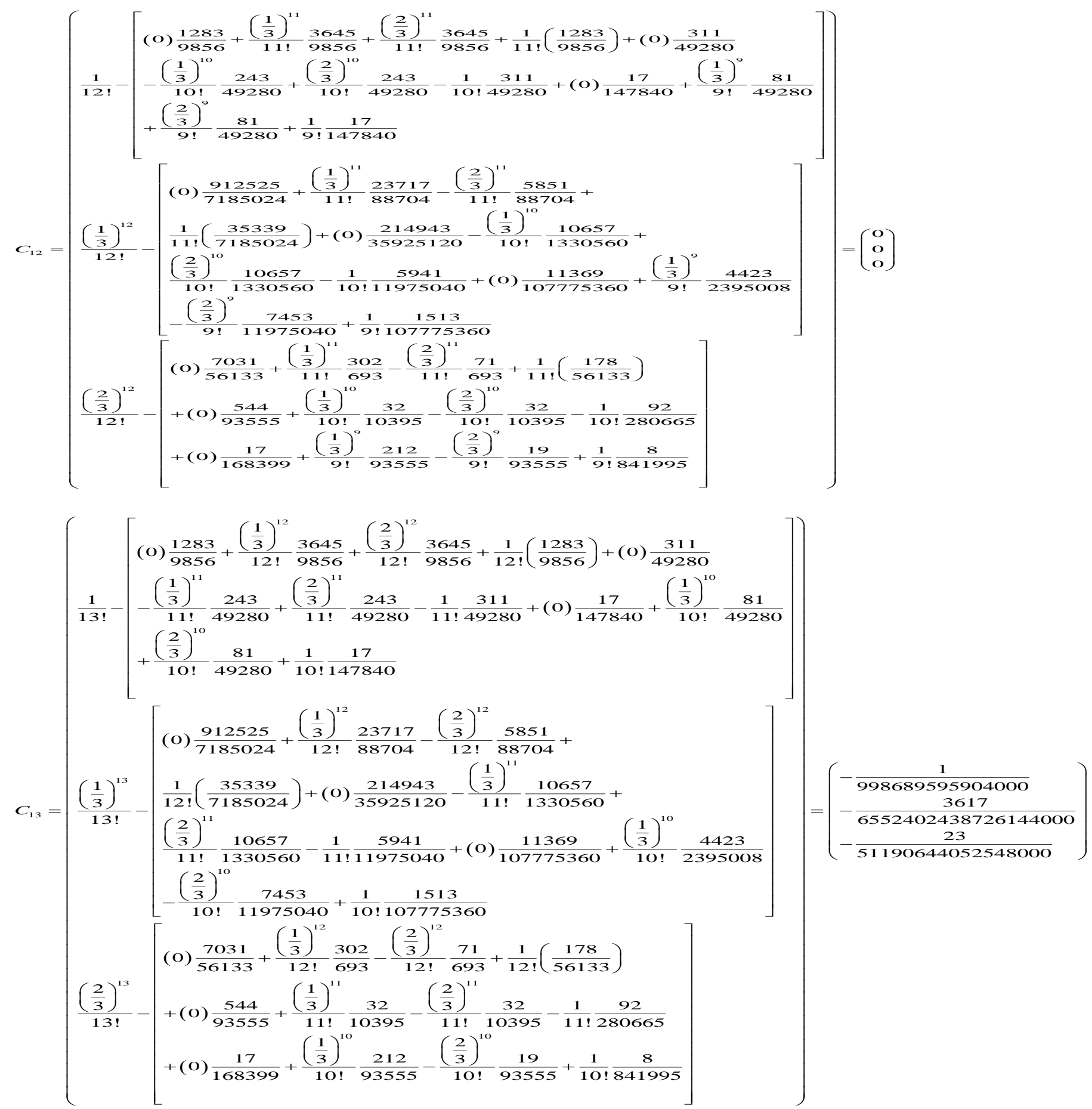

This implies that the block method has order $\mathrm{p}=12$ with error constant

$$
C_{13}=\left(-\frac{1}{998689595904000},-\frac{3617}{6552402438726144000},-\frac{23}{51190644052548000}\right)^{T}
$$

\subsection{Consistency and Zero Stability of OCB12 METHOD}

The linear multistep method is said to be consistent if it has order $p \geq 1$. Therefore, our method is consistent.

\section{Definition 3.3}


International Advanced Research Journal in Science, Engineering and Technology

Vol. 8, Issue 8, August 2021

DOI: $10.17148 /$ IARJSET.2021.8825

A hybrid Block method is said to be zero- stable if the roots $\mathrm{Z}$ of the characteristic polynomial $\bar{R}(Z)$ defined by:

$\bar{R}(Z)=\operatorname{det}\left[Z A^{0}-A^{\prime}\right]$

satisfies $|Z| \leq 1$ and every root with $\left|Z_{0}\right|=1$ has multiplicity not exceeding two in the Limit as $h \rightarrow 0$

Putting equation (6), (7) and (8) in matrix form as a block we obtain:

$$
\begin{aligned}
& {\left[\begin{array}{lll}
1 & 0 & 0 \\
0 & 1 & 0 \\
0 & 0 & 1
\end{array}\right]\left[\begin{array}{c}
y_{n+\frac{1}{3}} \\
y_{n+\frac{2}{3}} \\
y_{n+1}
\end{array}\right]=\left[\begin{array}{lll}
0 & 0 & 1 \\
0 & 0 & 1 \\
0 & 0 & 1
\end{array}\right]\left[\begin{array}{c}
y_{n-\frac{2}{3}} \\
y_{n-\frac{1}{3}} \\
y_{n}
\end{array}\right]} \\
& +h\left(\left[\begin{array}{ccc}
0 & 0 & \frac{1283}{9856} \\
0 & 0 & \frac{912523}{7185024} \\
0 & 0 & \frac{7031}{56133}
\end{array}\right]\left[\begin{array}{l}
f_{n-\frac{2}{3}} \\
f_{n-\frac{1}{3}} \\
f_{n}
\end{array}\right]+\left[\begin{array}{ccc}
\frac{3645}{9856} & \frac{3645}{9856} & \frac{1283}{9856} \\
\frac{23717}{88704} & -\frac{5851}{88704} & \frac{35339}{7185024} \\
\frac{302}{693} & \frac{71}{693} & \frac{178}{56133}
\end{array}\right]\left[\begin{array}{l}
f_{n+\frac{1}{3}} \\
f_{n+\frac{2}{3}} \\
f_{n+1}
\end{array}\right]\right)+ \\
& h^{2}\left(\left[\begin{array}{lll}
0 & 0 & \frac{311}{49280} \\
0 & 0 & \frac{214943}{35925120} \\
0 & 0 & \frac{544}{93555}
\end{array}\right]\left[\begin{array}{c}
g_{n-\frac{2}{3}} \\
g_{n-\frac{1}{3}} \\
g_{n}
\end{array}\right]\right)+\left[\begin{array}{lcc}
-\frac{243}{49280} & \frac{243}{49280} & -\frac{311}{49280} \\
-\frac{10657}{1330560} & \frac{10657}{1330560} & -\frac{5941}{11975040} \\
\frac{32}{10395} & -\frac{32}{10395} & -\frac{92}{280665}
\end{array}\right]\left[\begin{array}{c}
g_{n+\frac{1}{3}} \\
g_{n+\frac{2}{3}} \\
g_{n+1}
\end{array}\right] \\
& +h^{3}\left(\left[\begin{array}{lll}
0 & 0 & \frac{17}{147840} \\
0 & 0 & \frac{11369}{107775360} \\
0 & 0 & \frac{17}{168399}
\end{array}\right]\left[\begin{array}{c}
p_{n-\frac{2}{3}} \\
p_{n-\frac{1}{3}} \\
p_{n}
\end{array}\right]\right)+\left[\begin{array}{ccc}
\frac{81}{49280} & \frac{81}{49280} & \frac{17}{147840} \\
\frac{4423}{2395008} & -\frac{7453}{11975040} & \frac{1513}{107775360} \\
\frac{212}{93555} & -\frac{19}{93555} & \frac{8}{841995}
\end{array}\right]\left[\begin{array}{c}
p_{n+\frac{1}{3}} \\
p_{n+\frac{2}{3}} \\
p_{n+1}
\end{array}\right]
\end{aligned}
$$

The following matrix difference equation will be in form of:

$$
A^{0} y_{n+1}=A^{(')} y_{n-1}+h\left[B^{(')} f_{n-1}+B^{\prime} f_{n}\right]+h^{2}\left[C^{(')} g_{n-1}+C^{\prime} g_{n}\right]+h^{3}\left[D^{(')} p_{n-1}+D^{\prime} p_{n}\right]
$$

The first characteristics polynomial of the above matrix is given by

$$
\begin{aligned}
& R(Z)=\operatorname{det}\left[Z A^{0}-A^{\prime}\right] \\
& A^{0}=\left[\begin{array}{lll}
1 & 0 & 0 \\
0 & 1 & 0 \\
0 & 0 & 1
\end{array}\right], A^{\prime}=\left[\begin{array}{lll}
0 & 0 & 1 \\
0 & 0 & 1 \\
0 & 0 & 1
\end{array}\right]
\end{aligned}
$$


$R(Z)=\operatorname{det}\left[Z\left[\begin{array}{lll}1 & 0 & 0 \\ 0 & 1 & 0 \\ 0 & 0 & 1\end{array}\right]-\left[\begin{array}{lll}0 & 0 & 1 \\ 0 & 0 & 1 \\ 0 & 0 & 1\end{array}\right]\right]$

$=\operatorname{det}\left[\left[\begin{array}{lll}Z & 0 & 0 \\ 0 & Z & 0 \\ 0 & 0 & Z\end{array}\right]-\left[\begin{array}{lll}0 & 0 & 1 \\ 0 & 0 & 1 \\ 0 & 0 & 1\end{array}\right]\right]=\operatorname{det}\left[\begin{array}{lll}Z & 0 & -1 \\ 0 & Z & -1 \\ 0 & 0 & Z-1\end{array}\right]$

$R(Z)=Z^{2}(z-1)$

$R(Z)=0$

then the roots of $R(Z)=0$ satisfy $R(Z) \leq 1$

Hence, the block method is said to be zero-stable. Therefore, since the block method is consistent and zero-stable, it is likewise convergent.

\subsection{IMPLEMENTATION OF OCB12 METHOD.}

In this section we solve some tested IVPs with Maple software to determine the efficiency and usability of OCB12 method with other existing methods.

\section{Problem 1: [10]}

$y^{\prime}=0.5(1-y), y(0)=0.5, h=0.1$ with exact solution $y(x)=1-0.5 e^{-0.5 x}$

Problem 2: [1]

Problem 3: [14]

$$
y^{\prime}=-y, y(0)=1, h=0.1 \text { with exact solution } y(x)=e^{-x}
$$

$$
y^{\prime}=y, y(0)=1, h=0.1 \text { with exact solution } y(x)=e^{x}
$$

Table1. Comparison of computed results for problem 1

\begin{tabular}{|l|l|l|l|l|}
\hline $\mathrm{X}$ & Exact solution & $\begin{array}{l}\text { Computed solution in } \\
\text { (Zurni, et al.,2016) }\end{array}$ & $\begin{array}{l}\text { Computed solution in } \\
\text { (Sunday } \text { et al., 2013) }\end{array}$ & $\begin{array}{l}\text { Computed solution in } \\
\text { OCB12 Method }\end{array}$ \\
\hline 0.1 & 0.52438528774964299546 & 0.52438528774960472804 & 0.5243852877552174 & 0.52438528774964299545 \\
\hline 0.2 & 0.54758129098202021342 & 0.54758129098194536511 & 0.5475812909859664 & 0.54758129098202021341 \\
\hline 0.3 & 0.56964601178747109638 & 0.56964601178736527269 & 0.5696460117956543 & 0.56964601178747109637 \\
\hline 0.4 & 0.59063462346100907066 & 0.59063462346087361956 & 0.5906346234953703 & 0.59063462346100907065 \\
\hline 0.5 & 0.61059960846429756588 & 0.61059960846413739010 & 0.6105996086572718 & 0.61059960846429756586 \\
\hline 0.6 & 0.62959088965914106696 & 0.62959088965895722513 & 0.6295908898470451 & 0.62959088965914106694 \\
\hline 0.7 & 0.64765595514064328282 & 0.64765595514044005788 & 0.6476559553183269 & 0.64765595514064328279 \\
\hline 0.8 & 0.66483997698218034963 & 0.66483997698195855368 & 0.6648399771546479 & 0.66483997698218034959 \\
\hline 0.9 & 0.68118592418911335343 & 0.68118592418887672320 & 0.6811859243738679 & 0.68118592418911335339 \\
\hline 1.0 & 0.69673467014368328820 & 0.69673467014343242661 & 0.6967346704442603 & 0.69673467014368328816 \\
\hline
\end{tabular}

Table1b. Comparison of ERROR for problem 1 
International Advanced Research Journal in Science, Engineering and Technology

Vol. 8, Issue 8, August 2021

DOI: $10.17148 /$ IARJSET.2021.8825

\begin{tabular}{|c|c|c|c|}
\hline $\mathrm{X}$ & $\begin{array}{l}\text { Error in } \\
\text { (Zurni et al.,2016) }\end{array}$ & $\begin{array}{l}\text { Error in } \\
\text { (Sunday et al., } \\
\text { 2013) }\end{array}$ & $\begin{array}{l}\text { Error in } \\
\text { OCB12 Method }\end{array}$ \\
\hline 0.1 & $3.826740 \mathrm{E}-14$ & $5.574430 \mathrm{E}-12$ & $1.10^{-20}$ \\
\hline 0.2 & 7.484830E-14 & $3.946177 \mathrm{E}-12$ & $1.10^{-20}$ \\
\hline 0.3 & $1.058240 \mathrm{E}-13$ & $8.183232 \mathrm{E}-12$ & $1.10^{-20}$ \\
\hline 0.4 & $1.354510 \mathrm{E}-13$ & $3.436118 \mathrm{E}-11$ & $1.10^{-20}$ \\
\hline 0.5 & $1.601760 \mathrm{E}-13$ & $1.929473 \mathrm{E}-10$ & $2.10^{-20}$ \\
\hline 0.6 & $1.838420 \mathrm{E}-13$ & $1.879040 \mathrm{E}-10$ & 2. $10^{-20}$ \\
\hline 0.7 & $2.032250 \mathrm{E}-13$ & $1.776835 \mathrm{E}-10$ & $3.10^{-20}$ \\
\hline 0.8 & $2.217960 \mathrm{E}-13$ & $1.724676 \mathrm{E}-10$ & 4. $10^{-20}$ \\
\hline 0.9 & $2.366300 \mathrm{E}-13$ & $1.847545 \mathrm{E}-10$ & 4. $10^{-20}$ \\
\hline 1.0 & $2.508620 \mathrm{E}-13$ & $3.005770 \mathrm{E}-10$ & 4. $10^{-20}$ \\
\hline
\end{tabular}

Table 2. Comparison of computed results for problem 2

\begin{tabular}{|l|l|l|l|l|l|}
\hline $\mathrm{x}$ & Exact solution & $\begin{array}{l}\text { Computed solution in } \\
\text { (Badmus } \text { et al., 2015) }\end{array}$ & $\begin{array}{l}\text { Computed solution in } \\
\text { OCB12 Method }\end{array}$ & $\begin{array}{l}\text { Error in } \\
\text { (Badmus, et } \\
\text { al., 2015) }\end{array}$ & $\begin{array}{l}\text { ECB12 in } \\
\text { Method }\end{array}$ \\
\hline 0.1 & 0.90483741803595957316 & 0.904837417881202 & 0.90483741803595957321 & $1.5476 \mathrm{E}-10$ & $5.10^{-20}$ \\
\hline 0.2 & 0.81873075307798185867 & 0.818730752939751 & 0.81873075307798185875 & $1.3823 \mathrm{E}-10$ & $8.10^{-20}$ \\
\hline 0.3 & 0.74081822068171786607 & 0.740818220548903 & 0.74081822068171786618 & $1.3282 \mathrm{E}-10$ & $1.110^{-19}$ \\
\hline 0.4 & 0.67032004603563930074 & 0.670320045918305 & 0.67032004603563930088 & $1.1733 \mathrm{E}-10$ & $1.410^{-19}$ \\
\hline 0.5 & 0.60653065971263342360 & 0.606530659599218 & 0.60653065971263342376 & $1.1342 \mathrm{E}-10$ & $1.610^{-19}$ \\
\hline 0.6 & 0.54881163609402643263 & 0.548811635994641 & 0.54881163609402643280 & $9.9385 \mathrm{E}-11$ & $1.710^{-19}$ \\
\hline
\end{tabular}


International Advanced Research Journal in Science, Engineering and Technology

Vol. 8, Issue 8, August 2021

DOI: $10.17148 /$ IARJSET.2021.8825

\begin{tabular}{|l|l|l|l|l|l|}
\hline 0.7 & 0.49658530379140951470 & 0.496585303694640 & 0.49658530379140951489 & $9.6770 \mathrm{E}-11$ & $1.910^{-19}$ \\
\hline 0.8 & 0.4493289641172215914 & 0.449328964033219 & 0.44932896411722159162 & $8.4003 \mathrm{E}-11$ & $1.910^{-19}$ \\
\hline 0.9 & 0.40656965974059911188 & 0.406569659658082 & 0.40656965974059911208 & $8.2517 \mathrm{E}-11$ & $2.010^{-19}$ \\
\hline 1.0 & 0.36787944117144232160 & 0.367879441100594 & 0.36787944117144232179 & $7.0848 \mathrm{E}-11$ & $1.910^{-19}$ \\
\hline
\end{tabular}

Table 3a. Comparison of computed results and error for problem 3

\begin{tabular}{|l|l|l|l|l|l|}
\hline$x$ & Exact solution & $\begin{array}{l}\text { Computed solution } \\
\text { In [14] }\end{array}$ & $\begin{array}{l}\text { Computed solution in } \\
\text { OCB12 Method }\end{array}$ & $\begin{array}{l}\text { Error } \\
\text { In [14] }\end{array}$ & $\begin{array}{l}\text { Error In } \\
\text { OCB12 } \\
\text { METHOD }\end{array}$ \\
\hline 0.1 & 1.105170918075648 & 1.105170917860730 & 1.1051709180756476248 & $2.149179 \mathrm{E}-10$ & 0.00 \\
\hline 0.2 & 1.221402758160170 & 1.221402757685120 & 1.2214027581601698339 & $4.7505 \mathrm{E}-10$ & 0.00 \\
\hline 0.3 & 1.349858807576003 & 1.349858806788490 & 1.3498588075760031039 & $7.875129 \mathrm{E}-10$ & $1.10^{-19}$ \\
\hline 0.4 & 1.491824697641270 & 1.491824696480820 & 1.4918246976412703177 & $1.16045 \mathrm{E}-09$ & $1.10^{-19}$ \\
\hline 0.5 & 1.648721270700128 & 1.648721269097010 & 1.6487212707001281467 & $1.603118 \mathrm{E}-09$ & $1.10^{-19}$ \\
\hline 0.6 & 1.822118800390509 & 1.822118798264440 & 1.8221188003905089747 & $2.126069 \mathrm{E}-09$ & $2.10^{-19}$ \\
\hline 0.7 & 2.013752707470477 & 2.013752704729200 & 2.0137527074704765214 & $2.741277 \mathrm{E}-09$ & $2.10^{-19}$ \\
\hline 0.8 & 2.225540928492468 & 2.225540925030090 & 2.2255409284924676043 & $5.989459 \mathrm{E}-09$ & $3.10^{-19}$ \\
\hline 0.9 & 2.459603111156950 & 2.459603106852120 & 2.4596031111569496635 & $4.30483-09$ & $3.10^{-19}$ \\
\hline 1.0 & 2.718281828459046 & 2.718281824122030 & 2.7182818284590452350 & $4.337016 \mathrm{E}-09$ & $4.10^{-19}$ \\
\hline
\end{tabular}

\section{CONCLUSION}

In this paper, we have presented an implicit one-step third Derivative hybrid Block Method for Solution of first order initial value problems in ordinary differential equations. The property of the method has shown its convergence and the numerical problems considered have likewise shown its superiority over previously existing methods in literature.

\section{REFERENCES}

[1]. Sunday, J., Odekunle, M. R. , Adesanya, A. O. and James, A. A. (2013).: Extended Block Integrator for First-Order Stiff and Oscillatory Differential Equations, American Journal of Computational and Applied Mathematics, 3(6): 283-290.

[2]. Nur, Zahidah Mukhtar and Zanariah, Abdul Majid ( 2015): Extended One-Step Block Method for Solving Stiff Initial Value Problem. 7th International Conference On Research And Educations In Mathematics (ICREM7). DOI: 10.1109/ICREM.2015.7357040.

[3]. Timothy A.A, David O. A, and Adetola .A. A. (2012): A One Step Method for the Solution of General Second Order Ordinary Differential Equations. International Journal of Science and Technology, Volume 2 No.4.

[4]. W.E. Milne, Numerical Solution of Differential Equations, John Wiley and Sons, 1953.

[5]. Shampine,. L.F and Watts, H. A(1969) : Block implicit one-step methods. Mathematics of Computation, 23, no. 108, 731-740.

[6]. Omar, .Z and Alkasassbeh, M. F(2016): Generalized One-Step Third Derivative Implicit Hybrid Block Method for the Direct Solution of Second Order Ordinary Differential Equation. Applied Mathematical Sciences, Vol. 10, no. 9, 417 - 430

[7]. Gear, C.W.( (1965): Hybrid methods for initial value problems in ordinary differential equations, SIAM Journal of Numerical Analysis, 2 no. $1,69-86$.

[8]. Bothayna, S.H. Kashkari, Muhammed I. Syam (2019): Optimization of one step block method with three hybrid points for solving first-order ordinary differential equations, journal homepage:www.elsevier.com/locate/rinp. Results in Physics 12, 592-596

[9] Areo, E. A And Adeniyi, R.B.(2014). Block Implicit One-Step Method for The Numerical Integration of Initial Value Problems In Ordinary Differential Equations. International Journal of Mathematics and Statistics Studies Vol.2, No.3, Pp.4-13.

[10]. Ali, Shokri (2014), : One and two-step new hybrid methods for the numerical solution of first order initial value problems, Acta Universitatis Matthiae Belii, series Mathematics Volume 22,pp 45-58, ISSN 1338-712X, ISBN 978-80-557-0812-6

[11]. Yakusak, N. S. and Adeniyi, R. B. (2015). A Four-Step Hybrid Block Method for First Order Initial Value Problems in Ordinary Differential Equations. AMSE JOURNALS -2015-Series: Advances A; Vol. 52; No 1; pp 17-30 


\section{International Advanced Research Journal in Science, Engineering and Technology}

Vol. 8, Issue 8, August 2021

\section{DOI: 10.17148/IARJSET.2021.8825}

[12] Badmus, A.M., Yahaya, Y.A. and Pam, Y.C.(2015). Adams type hybrid block methods associated with chebyshev polynomial for the solution of ordinary differential equations. British J. Math. Comput. Sci., 6: 464-474.

[13]. Zurni, Omar and Oluwaseun, adeyeye (2016). Numerical solution of first order initial value problems using a self-starting implicit two-step obrechkoff-type block method. Journal of mathematics and statistics, 12(2): 27-134. DOI: 10.3844/jmssp.2016.127.134

[14]. Ajileye, G, Amoo, S.A and Ogwumu, O.D.(2018). Hybrid Block Method Algorithms for Solution of First Order Initial Value Problems in Ordinary Differential Equations. Journal of Applied \& Computational Mathematics, volume 7, issue 2, DOI: 10.4172/2168-9679.1000390

[15].Zurni Omar and Oluwaseun adeyeye(2016). Numerical solution of first order initial value problemsusing a self-starting implicit two-step obrechkoff-type block method. Journal of mathematics and statistics, 12(2): 27-134. 